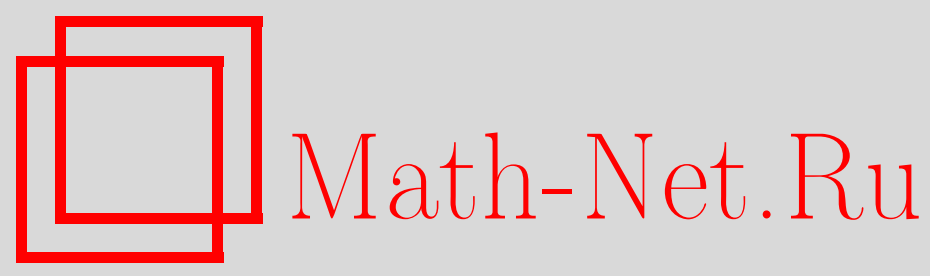

И. В. Изместьев, Трехмерные многообразия, определяемые раскраской граней простого многогранника, Матем. заметки, 2001, том 69, выпуск 3, 375-382

DOI: https://doi.org/10.4213/mzm511

Использование Общероссийского математического портала Math-Net.Ru подразумевает, что вы прочитали и согласны с пользовательским соглашением http://www . mathnet.ru/rus/agreement

Параметры загрузки:

IP : 35.173 .137 .237

26 апреля 2023 г., 16:38:02

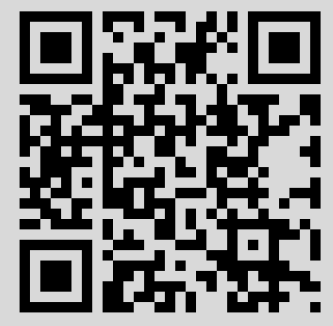




\section{ТРЕХМЕРНЫЕ МНОГООБРАЗИЯ, ОПРЕДЕЛЯЕМЫЕ РАСКРАСКОЙ ГРАНЕЙ ПРОСТОГО МНОГОГРАННИКА}

\section{И.В. Изместьев}

В статье вводится и изучается некоторый класс трехмерных многообразий с действием группы $\mathbb{Z}_{2}^{3}$ и простьм выпуклым многогранником в качестве пространства орбит. Эти многообразия возникают из трехмерных многогранников, грани которых допускают правильную раскраску в три цвета, при помощи конструкции, используемой при изучении квазиторических многообразий. Для многообразий рассматриваемого вида доказано существование эквивариантного вложения в пространство $\mathbb{R}^{4}$. Также описано действие на их множестве операций эквивариантной связной суммы и эквивариантной перестройки Дена. Доказано, что любое такое многообразие получается из конечного числа трехмерных торов с каноническим действием группы $\mathbb{Z}_{2}^{3}$ путем применения указанных операций.

Библиография: 7 названий.

1. Введение и основные определения. Пусть $P$ - простой вьпукльй многогранник, имеющий $m$ гиперграней, которые мы произвольным образом упорядочим: $F_{1}, \ldots, F_{m}$. Пусть $G$ обозначает одну из груп $\mathbb{Z}_{2}$ или $\mathbb{S}^{1}$. Через $G_{i}$ мы будем обозначать $i$-ю координатную подгруппу группы $G^{m}$.

ОПРЕДЕЛЕНиЕ 1 (см. [1], [2]). Введем на декартовом произведении $G^{m} \times P$ отношение эквивалентности

$$
(g, p) \sim\left(g^{\prime}, p^{\prime}\right) \Longleftrightarrow p^{\prime}=p, g^{\prime} g^{-1} \in \bigoplus_{F_{i} \ni p} G_{i} .
$$

Тогда пространство, определяемое многогранником $P$, это

$$
\mathscr{Z}_{P}=\left(G^{m} \times P\right) / \sim \text {. }
$$

Рассмотрение локального устройства факторпространства (2) показывает, что $\mathscr{Z}_{P}-$ многообразие. На нем имеется естественное действие групшы $G^{m}$, заданное формулой $h[g, p]=[h g, p]$, при этом пространством орбит является многогранник $P$.

Ряд интересных многообразий возникает из многообразия $\mathscr{Z}_{P}$ путем применения следующей конструкции. Пусть имеется мономорфизм $\iota: G^{k} \rightarrow G^{m}$ такой, что определяемое им действие $h[g, p]=[\iota(h) g, p]$ групшы $G^{k}$ на многообразии $\mathscr{Z}_{P}$ свободно. Тогда однородное пространство $\mathscr{Z}_{P} / G^{k}$ также является многообразием, на котором действует уже группа $G^{m-k} \cong G^{m} / \iota\left(G^{k}\right)$ по-прежнему с пространством орбит - многогранником $P$. Обозначим максимальное $k$, при котором существует такое свободное действие $G^{k}$ на $\mathscr{Z}_{P}$, через $k^{*}(P)$. В.М. Бухштабером предложено рассматривать число $k^{*}(P)$ 
как новьй комбинаторньй инвариант простого вьпуклого многогранника $P$. Можно показать, что $1 \leqslant k^{*}(P) \leqslant m-n$, где $n$ - размерность многогранника $P$. Особый интерес представляют многогранники $P$ с $k^{*}(P)=m-n$. В этом случае при $G=\mathbb{S}^{1}$ возникает многообразие $M^{2 n}=\mathscr{Z}_{P} / \mathbb{T}^{m-n}$, которое назьвается квазиторическим. Эти многообразия замечательны во многих отношениях (см. [1]).

Нас будет интересовать следующий частньй случай конструкции, описанной в предыдущем абзаце. Рассмотрим сюръективное отображение $\chi:\{1, \ldots, m\} \rightarrow\{1, \ldots, s\}$. Понему строится эпиморфизм $\widetilde{\chi}: G^{m} \rightarrow G^{s}$, удовлетворяющий условию $\widetilde{\chi}\left(G_{i}\right)=G_{\chi(i)}$. Возьмем любой мономорфизм $G^{m-s} \rightarrow G^{m}$, образ которого совпадает с ядром $\widetilde{\chi}$. Нетрудно доказать, что определяемое им действие группы $G^{m-s}$ на многообразии $\mathscr{Z}_{P}$ свободно в том и только том случае, если $F_{i} \cap F_{j} \neq \varnothing$ влечет $\chi(i) \neq \chi(j)$ для любых двух различных гиперграней многогранника $P$. Соответствующее отображение $F_{i} \mapsto \chi(i)$ назьвается в этом случае правильной раскраской множества гиперграней в $s$ цветов. Таким образом, можно говорить о многообразии $\mathscr{Z}(P, \chi)$, определяемом раскраской. Этот прием позволяет следующим образом оценить число $k^{*}$ снизу.

В теории графов вершинным хроматическим числом $\gamma(K)$ графа $K$ назьвается наименьшее число цветов, в которое можно покрасить его вершины так, чтобы любая пара смежных вершин имела различные цвета. Тогда имеем

$$
k^{*}(P) \geqslant m-\gamma\left(K_{1}(P)\right),
$$

где $K_{1}(P)$ - граф смежности гиперграней многогранника $P$.

Многообразие $\mathscr{Z}(P, \chi)$ можно описать более явно как факторпространство декартова произведения $G^{s} \times P$ по отношению эквивалентности $(1)$, в котором вместо условия $g^{\prime} g^{-1} \in \bigoplus_{F_{i} \ni p} G_{i}$ фигурирует условие $g^{\prime} g^{-1} \in \widetilde{\chi}\left(\bigoplus_{F_{i} \ni p} G_{i}\right)$. Последнее же, как нетрудно видеть, равносильно тому, что $g^{\prime} g^{-1} \in \bigoplus_{F_{i} \ni p} G_{\chi(i)}$. Таким образом, мы приходим к следующему определению.

ОПРЕДЕЛЕНИЕ 2 . Пусть $\chi:\{1, \ldots, m\} \rightarrow\{1, \ldots, s\}-$ сюръективное отображение, задающее правильную раскраску множества гиперграней простого выпуклого многогранника $P$ в $s$ цветов. Многообразием $\mathscr{Z}(P, \chi)$, определяемым раскраской $\chi$, называется факторпространство декартова произведения $G^{s} \times P$ по отношению эквивалентности

$$
(g, p) \sim\left(g^{\prime}, p^{\prime}\right) \Longleftrightarrow p^{\prime}=p, g^{\prime} g^{-1} \in H(p),
$$

где группа $H(p)$ определяется равенством

$$
H(p)=\bigoplus_{F_{i} \ni p} G_{\chi(i)} .
$$

В случае групшы $G=\mathbb{Z}_{2}$ построение пространства $\mathscr{Z}(P, \chi)$ можно описать как склейку $2^{s}$ экземпляров многогранника $P$ по некоторым парам граней одинакового цвета.

Мы будем заниматься случаем, когда группа $G$ равна $\mathbb{Z}_{2}$, а многогранник $P$ трехмерен и его грани покрашены в три цвета. Соответствующие многообразия также имеют размерность 3. В настоящей работе будет доказано существование эквивариантного вложения многообразия такого вида в четырехмерное евклидово пространство. Для них также будут определены эквивариантные перестройки Дена, и будет доказано, что класс таких многообразий порождается эквивариантньми связными суммами и перестройками Дена из трехмерньх торов. 
2. Эквивариантное вложение. Под многогранником мы всюду подразумеваем простой вьпукльй многогранник и, если не оговорено особо, трехмерньй. Многогранники, допускающие правильную раскраску граней в три цвета, характеризуются следуюшим свойством (см. [3, задача 42$])$.

Теорема 1. Грани многогранника $P$ могут быть правильно раскрашены в три ивета в том и только том случае, если каждая его грань содержит четное число ребер, т.е. реберный граф (он же одномерный остов) $P^{1}$ является двудольным графом. При этом раскраска единственна с точностью до смены цветов (перестановки әлементов множества $\{1,2,3\})$.

Таким образом, многообразия, определяемые различными раскрасками граней данного многогранника в три цвета, гомеоморфны друг другу эквивариантно относительно автоморфизма групшы $\mathbb{Z}_{2}^{3}$, переставляющего слагаемые. Поэтому мы можем рассматривать многообразие $\mathscr{Z}_{3}(P)$, определенное с точностью до автоморфизма действия.

ПримеР. Многообразие, определяемое кубом, - это трехмерный тор $\left(\mathbb{S}^{1}\right)^{3}$ со следующим действием групшы $\mathbb{Z}_{2}^{3}$. Каждая образующая действует осевой симметрией на соответствующую ей окружность, оставляя точки других окружностей неподвижными.

ТЕОрема 2. Для любого многогранника $P$, одномерный остов которого двудолен, определяемое им многообразие $\mathscr{Z}_{3}(P)$ эквивариантно вкладывается в евклидово пространство $\mathbb{R}^{4}$, на котором группа $\mathbb{Z}_{2}^{3}$ действует отражсениями относительно трех из четырех координатных гиперпространств.

ДокАЗАТЕЛЬСтво. Пространство орбит $\mathbb{R}^{4} / \mathbb{Z}_{2}^{3}$ по действию, описанному в условии теоремы, представляет собой произведение октанта $\mathbb{R}_{+}^{3}$ на прямую $\mathbb{R}$. Пространство $\mathbb{R}^{4}$ является результатом факторизации произведения $\mathbb{Z}_{2}^{3} \times\left(\mathbb{R}_{+}^{3} \times \mathbb{R}\right)$ по отношению эквивалентности (3), где групшы $H(p)$ определяются раскраской граней октанта по тому же принципу, что и раньше. Таким образом, если мы вложим многогранник $P$ в пространство $\mathbb{R}_{+}^{3} \times \mathbb{R}$ с условием, что образ грани данного цвета лежит в множестве того же цвета, то после применения описанной конструкции к пространству $\mathbb{R}_{+}^{3} \times \mathbb{R}$ получим эквивариантное вложение $\mathscr{Z}_{3}(P) \rightarrow \mathbb{R}^{4}$.

Требуемое вложение $P \rightarrow \mathbb{R}_{+}^{3} \times \mathbb{R}$ мы будем строить, начиная с одномерного остова $P^{1}$ многогранника $P$. Правильная раскраска граней многогранника $P$ порождает правильную раскраску ребер графа $P^{1}$ по следующему правилу: ребро окрашивается в цвет, противоположный цветам граней, в которых оно содержится. Нетрудно видеть, что все ребра одного цвета должны вкладьваться в одну из полуплоскостей, являющихся произведением координатных полуосей октанта $\mathbb{R}_{+}^{3}$ на прямую $\mathbb{R}$. При этом граф $P^{1}$ вкладывается в книжку с тремя страницами $\left(\mathbb{R}_{+} \vee \mathbb{R}_{+} \vee \mathbb{R}_{+}\right) \times \mathbb{R}$, его вершины попадают на ось $\{0\} \times \mathbb{R}$.

Лемма 1. Граф $P^{1}$, ребра которого покрашены в соответствии с описанным выше правилом, можно вложить в книжку с тремя страницами тех же иветов так, чтобы каждое ребро попало на страницу одного с ним цвета.

ДокАЗАТЕЛЬСтво. Легко заметить, что построение такого вложения равносильно расположению вершин граффа $P^{1}$ на прямой с выполнением условия: пары вершин, соединенные ребрами одного цвета, не должны разделять друг друга. С тем же успехом 
вершины можно располагать на окружности. В этой последней формулировке мы и будем доказывать лемму.

Рассмотрим двумерный остов многогранника как сферу с вложенньм в нееграфом $P^{1}$. Для простоты дальнейших построений будем считать, что ребра графа являются дугами больших окружностей. Это достигается центральным проектированием из некоторой точки внутри многогранника на сферу с центром в этой точке. Мы построим на сфере простую замкнутую кривую $C$, проходящую через все вершины $P^{1}$ так, чтобы она не разделяла ребра одного цвета (т.е. чтобы все ребра одного цвета лежали в одной из двух областей, на которые $C$ делит сферу). Соответствующее расположение вершин на окружности, гомеоморфным образом которой является $C$, как нетрудно видеть, будет удовлетворять требуемому условию.

Пусть $\left\{F_{i}\right\}_{i=1}^{k}$ - все грани цвета 3 (рассматриваемые сейчас как подмножества сферы). Включим каждую грань $F_{i}$ в диск $D_{i}$ так, чтобы вершины грани лежали на гранище диска, а внутренности ее ребер - в его внутренности. Границу диска $D_{i}$ можно составить из дуг окружностей с концами в вершинах грани $F_{i}$. Потребуем также, чтобы диски не пересекались. Рассмотрим граб с множеством вершин $\left\{D_{i}\right\}$ и ребрами, соответствуюшими ребрам цвета 1 и 2 графа $P^{1}$. Его можно получить путем стягивания всех дисков $D_{i}$ в точки, причем допускаются кратные ребра. Выберем в этом графе какое-либо максимальное дерево. Для каждого ребра, входящего в дерево, рассмотрим соответствующее ребро е графа $P^{1}$. Оно соединяет какие-то два из дисков $D_{i}$. Проведем в малой окрестности ребра $e$ путь, его не пересекающий, и рассмотрим еще более малое утолщение этого пути. Очевидно, все это можно проделать так, что объединение дисков $D_{i}$ с построенньми утолщениями будет гомеоморфно диску и будет содержать внутри себя только ребра цвета 3. Граница этого диска является простой замкнутой кривой, отделяющей ребра цвета 3 от ребер цветов 1 и 2 ; следовательно, ее можно взять в качестве искомой. Лемма доказана.

Теперь нам требуется продолжить полученное вложение $P^{1} \rightarrow \mathbb{R}_{+}^{3} \times \mathbb{R}$ на грани и внутренность многогранника. Все грани одного цвета должны быть вложены в пространство $\mathbb{R}_{+}^{2} \times \mathbb{R}$, ограниченное двумя из трех страниц книжки, на которых уже лежат их границы. Поскольку эти гранищы не пересекаются, требуемое продолжение возможно. После этого остается продолжить вложение на внутренность $P$, что тоже не составляет труда, поскольку граница многогранника вложена в $\partial\left(\mathbb{R}_{+}^{3}\right) \times \mathbb{R}$, и мы можем взять конус над ней с вершиной во внутренней точке пространства $\mathbb{R}_{+}^{3} \times \mathbb{R}$. Теорема доказана.

СлЕдСТВИЕ 1. Любой 3-связный двудольный тривалентный әрафГС $\mathbb{S}^{2}$ можно представить как пересечение сферы с книжккой из трех странии при некотором вложении $\mathbb{S}^{2} \rightarrow \mathbb{R}^{3}$.

(3- связным назьвается граф, не теряющий связности при выбрасывании любых двух его вершин вместе со всеми выходяшими из них ребрами; тривалентным мы называем граф, из каждой вершины которого выходит три ребра.)

ДокАЗАТЕЛЬСтво. Теорема Штейница (см. [4, гл. 2, п. 15]) гласит, что любой 3-связный планарный граф является одномерным остовом некоторого вьпуклого многогранника. Следовательно, граф Г является остовом некоторого простого вьпуклого многогранника $P$, и при этом по теореме 1 грани $P$ могут быть правильно покрашены в три цвета. Но тогда требуемое вложение было построено на предпоследнем этапе доказательства теоремы 2. 
Заметим, что в следствии 1 условие 3-связности можно опустить; допустимо также наличие кратных ребер в графе Г. В этом случае доказательство проводится по образцу леммы 1 и следующих за ней рассуждений.

3. Эквивариантные перестройки. Определим две операции на множестве многогранников рассматриваемого вида. Первая из них, связная сумма, определяется для любых простых вьпуклых многогранников.

ОПРЕДЕЛЕНИЕ 3 (см. также [5]). Пусть $P_{1}$ и $P_{2}$ - произвольные простые выпуклые многогранники одинаковой размерности. Пусть в них выбраны вершины $v_{1}$ и $v_{2}$, и установлено взаимно однозначное соответствие между множеством гиперграней первого многогранника, смежных с вершиной $v_{1}$, и гиперграней второго, смежных с вершиной $v_{2}$. Связная сумма $P_{1} \# P_{2}$ по отношению к этим данньм определяется как многогранник, комбинаторно эквивалентньй результату склейки многогранников $P_{1}$ и $P_{2}$ по границам удаленных из них малых окрестностей вершин $v_{1}$ и $v_{2}$. При этой склейке соответствующие друг другу гиперграни многогранников должны объединяться в одну.

Это определение может быть обобщено на более широкий случай многообразий с углами. То, что связная сумма многогранников комбинаторно эквивалентна некоторому многограннику, доказывается построением, приведенным в [5]. В случае трехмерных многогранников можно воспользоваться теоремой Штейница: нетрудно видеть, что граф, получающийся таким образом из реберных графов многогранников $P_{1}$ и $P_{2}$, является 3-связным и планарным.

Заметим, что связная сумма трехмерных многогранников, имеющих двудольные одномерные остовы, снова обладает тем же свойством. Если при этом фиксирована правильная раскраска граней слагаемых в три цвета, то биекция граней, смежных с вершинами, в которых производится суммирование, возникает естественньм образом, и связная сумма наследует раскраску слагаемых. Поэтому корректно определена связная сумма $\left(P_{1}, \chi_{1}\right) \# v_{1}, v_{2}\left(P_{2}, \chi_{2}\right)$ трехмерных многогранников, грани которых правильно раскрашены в три цвета.

Вторая операция является перестройкой трехмерного многогранника и также не выводит за пределы многогранников рассматриваемого нами класса. Она действует в окрестности одного ребра, как изображено на рис. 1.
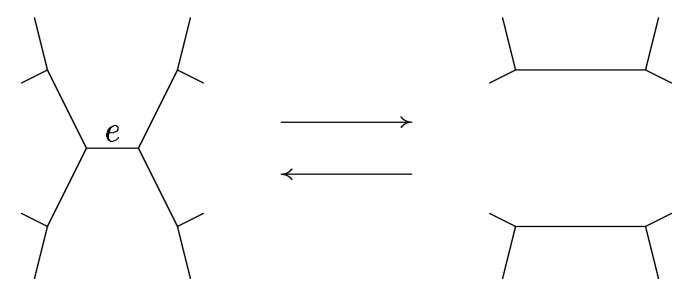

Рис. 1

Здесь показано действие перестройки и обратной к ней на одномерном остове многогранника. В том случае, если трехсвязность графа не нарушается, мы имеем корректно определенную перестройку многогранника (в силу теоремы Штейница).

Двум определенным выше операциям соответствуют операции на множестве многообразий, определяемых раскраской граней многогранника в три цвета. Связной сумме 
соответствует эквивариантная связная сумма многообразий, определенная в [1] в случае действия, устроенного локально некоторым стандартным образом. Заметим, что в многообразии $\mathscr{Z}(P, \chi)$ малая окрестность точки, соответствующей вершине многогранника $P$, эквивариантно гомеоморфна пространству $\mathbb{R}^{3}$ со стандартньм действием групाш $\mathbb{Z}_{2}^{3}$.

ОПРЕДЕЛЕНИЕ 4. Эквивариантная связная сумма $\mathscr{Z}\left(P_{1}, \chi_{1}\right) \#_{v_{1}, v_{2}} \mathscr{Z}\left(P_{2}, \chi_{2}\right)$ многообразий $\mathscr{Z}\left(P_{1}, \chi_{1}\right)$ и $\mathscr{Z}\left(P_{2}, \chi_{2}\right)$ относительно вериин $v_{1}$ и $v_{2}$ многогранников $P_{1}$ $u P_{2}$ - это результат эквивариантной склейки слагаемых по гранищам удаленных из них малых шаровых окрестностей точек, соответствующих вершинам $v_{1}$ и $v_{2}$.

Очевидна следующая лемма.

Лемма 2. Для любых многогранников $P_{1}$ и $P_{2}$ с раскрасками их граней $\chi_{1}$ и $\chi_{2}$ имеется әквивариантный гомеоморфизм

$$
\mathscr{Z}\left(P_{1}, \chi_{1}\right) \#_{v_{1}, v_{2}} \mathscr{Z}\left(P_{2}, \chi_{2}\right) \cong \mathscr{Z}\left(\left(P_{1}, \chi_{1}\right) \#_{v_{1}, v_{2}}\left(P_{2}, \chi_{2}\right)\right)
$$

Примечательно, что топологически эквивариантная связная сумма не зависит от вершин, в которых происходит суммирование. Действие группы при этом, как правило, различно, поскольку могут различаться пространства орбит.

Теперь мы займемся перестройками многообразия $\mathscr{Z}(P, \chi)$, соответствующим перестройкам многогранника, изображенным на рис. 1.

ОПРЕДЕЛЕНИЕ 5 (см. [6]). Перестройкой Дена трехмерного многообразия называется операция вырезания полнотория с приклейкой его затем на то же место по гомеоморфизму границы, меняющему местами меридианы и параллели.

Для полного описания перестройки Дена нужно указать окружность внутри многообразия, являющуюся осью вырезаемого полнотория.

Лемма 3. Перестройке многогранника $P$, изобраэкенной на рис. 1 (стрелка слева направо), соответствует перестройка Дена многообразия $\mathscr{Z}(P, \chi)$ вдоль окружности, возникающей из ребра е.

Во-первых, нетрудно видеть, что в многообразии $\mathscr{Z}(P, \chi)$ два экземпляра ребра $e$ действительно склеиваются в окружность. Перестройку многогранника можно представить как вырезание четверти цилиндра в окрестности данного ребра и приклейку на это место половины цилиндра, как это показано на рис. 2.

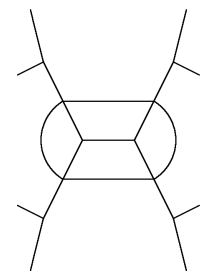

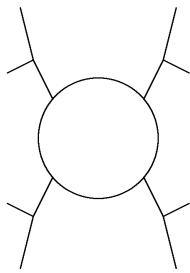

Рис. 2

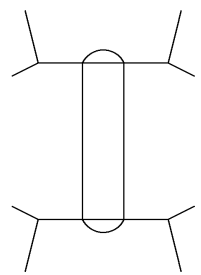


Рассмотрев, какие подмногообразия в $\mathscr{Z}(P, \chi)$ отвечают вырезаемой и вклеиваемой частям, мы обнаружим, что оба они являются полноториями, причем меридианы и параллели одного из них соответствуют параллелям и меридианам другого. Таким образом, им отвечают взаимнообратные перестройки Дена многообразия, определяемого раскраской. Поскольку действие группы на “прооперированном" многообразии определено канонически, мы назовем эти перестройки эквивариантными перестройками Дена для многообразий рассматриваемого нами вида.

Как показано в [7], любое трехмерное многообразие можно получить из трехмерной сферы последовательным применением перестроек Дена. Основным результатом этой части будет доказательство аналогичного факта о порождении всех многообразий, определяемых раскраской граней трехмерного многообразия в три цвета.

Теорема 3. Каждое многообразие вида $\mathscr{Z}(P, \chi)$ может быть получено из набора трехмерных торов ( действием, описанным в примере п. 2) путем применения конечного числа операций связной суммы и әквивариантной перестройки Дена.

ДокАЗАТЕЛЬСТво. Достаточно доказать, что любой простой многогранник с двудольньм реберньм графом можно получить из набора кубов при помощи описанных выше операций на многогранниках; или наоборот, перестройками Дена и разложениями в связную сумму можно привести любой многогранник к набору кубов.

Нам пригодятся следующие несложные критерии.

ЛЕмма 4. 1) Тривалентный граф $Г \subset S^{2}$ тогда и только тогда не является 3-связным, когда существует простая замкнутая кривая $C \subset S^{2}$, не содерэсащая вершин графа и пересекающая не более двух его ребер при том, что каждая из двух компонент $S^{2} \backslash C$ содерэит по крайней мере одну вериину Г.

2) Многогранник, допускающий правильную раскраску граней в три ивета и имеющий реберный граф $Г \subset S^{2}$, тогда и только тогда распадается в связную сумму многогранников с тем же свойством, когда существует простая замкнутая кривая $C \subset S^{2}$, не содерэсащая вериин графа Г и пересекающая не более трех его ребер при том, что каждая из двух компонент $S^{2} \backslash C$ содержит по крайней мере две вериины Г.

\section{ДоКАЗАТЕЛЬСТВо несложно и оставляется читателю.}

В первой части доказательства теоремы мы покажем, как при помощи перестроек Дена и разложений в связную сумму привести данный многогранник к набору призм. Пусть $Г \subset S^{2}$ - одномерньй остов многогранника. Области, на которые $Г$ разбивает $S^{2}$, отождествляются с гранями. Рассмотрим множество граней, состояших не менее, чем из шести ребер. Будем называть такиеграни большими. Если никакие две большие грани не смежны, то многогранник является призмой. Поэтому будем считать, что среди больших граней есть соседние. Заметим также, что по соображениям эйлеровой характеристики все грани большими быть не могут. Из этого вытекает, что найдется вершина, в которой сходятся две большие грани $(A$ и $B)$ и один четырехугольник $(C)$. Произведем перестройку рис. 1 для общего ребра граней $A$ и $B$. Если полученньй граф $\Gamma^{\prime}$ останется 3-связным, то он будет каркасом многогранника с числом граней, на единицу меньшим. Предположим, что 3-связность нарушается, и докажем, что в этом случае исходный многогранник можно представить в виде связной суммы. Из утверждения 1) 
леммы вытекает, что какие-то две грани $\Gamma^{\prime}$ имеют два общих ребра. Одной из них, очевидно, является грань, образующаяся в результате слияния $C$ со второй общей смежной к $A$ и $B$ гранью. Вторую грань обозначим $D$. Пусть $e$ и $f$ - два общих ребра этих граней. Тогда (см. рис. 3) разрезание ребер $f, g$ и $h$ нарушает связность графа Г. При этом, так как грань $A$ большая, обе части, возникающие в результате разрезания, содержат более двух вершин. Следовательно, по утверждению 2) леммы граф Г распадается в связную сумму. Продолжая этот процесс, мы на каждом шаге будем получать многогранники с меньшим числом граней, поэтому рано или поздно придем к набору призм.

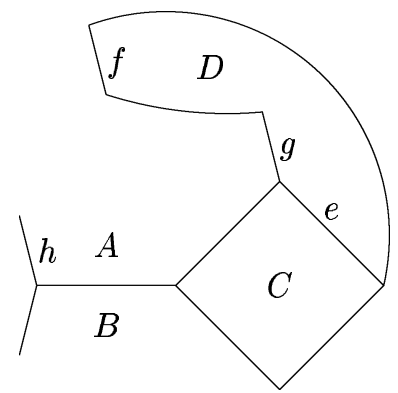

Рис. 3

Теперь рассмотрим призму, в основании которой лежит $2 n$-угольник, $n \geqslant 3$. Мы можем склеить два ребра (стрелка в левую сторону на рис. 1) основания призмы, между которыми заключены два других ребра. После этого многогранник разлагается в прямую сумму куба и $(2 n-2)$-угольной призмы. Таким образом, призма приводится к набору кубов, что завершает доказательство теоремы.

Автор благодарит своего научного руководителя профессора В. М. Бухштабера за внимание к работе и ценные замечания по ее содержанию.

\section{СПИСОК ЦИТИРОВАННОЙ ЛИТЕРАТУРЫ}

[1] Davis M.W., Januszkiewicz T. Convex polytopes, Coxeter orbifolds and torus actions // Duke Math. J. 1991. V. 62. № 2. P. 417-451.

[2] Бухштабер В. М., Панов Т. Е. Действия тора и многообразия, определяемые простьми многогранниками // Тр. МИАН. 1999. Т. 225. С. 96-131.

[3] Дынкин Е. Б., Успенский В. А. Математические беседы. М.-Л.: ГИТТЛ, 1952.

[4] Бренстед А. Введение в теорию выпуклых многогранников. М.: Мир, 1988.

[5] Buchstaber V.M., Ray N. Tangential structures on toric manifolds, and connected sums of polytopes // UMIST. Manchester. 2000. V. 5.

[6] Прасолов В.В., Сосинский А. Б. Узлы, зацепления, косы и трехмерные многообразия. М.: МЦНМО, 1997.

[7] Kirby R. A calculus for framed links in $S^{3} / /$ Invent. Math. 1978. V. 45. P. 35-56.

Московский государственный университет им. М.В. Ломоносова

Поступило

E-mail: izmestiev@higeom.math.msu.su, izmest@mccme.ru 19.06.2000 